\title{
Estructura social y semántica: la lógica de una distinción sistémica
}

\author{
Social Structure and Semantic: The Logic of a Systemic Distinction
}

Rudolf Stichweh

Foro Internacional de Ciencia, Universidad de Bonn, Alemania

RESUMEN

El presente trabajo aborda de manera crítica la relación teórica entre estructura social y semántica, de modo de avanzar en una comprensión fructífera para las investigaciones. Se describen y analizan las diferentes concepciones de semántica y las relaciones de ésta con las estructuras sociales. Junto con lo anterior, se cuestiona la propuesta de una posible correlación entre ambos conceptos y se propone, en su lugar, una aproximación más cercana a la teoría de sistemas y la teoría de la evolución. El trabajo concluye con una propuesta para la distinción entre estructura social y semántica, en la cual se destaca la posibilidad que la sociedad describa sus estructuras posibles mediante su propia semántica. Este enfoque haría posible una comprensión de la semántica en un sentido anticipativo, reconstructivo y constitutivo respecto de las estructuras sociales.

PALABRAS ClAVE: Estructura social; Semántica; Sistemas sociales; Diferenciación funcional; Historia de las ideas

\section{ABSTRACT}

The present paper addresses the theoretical link between social structure and semantic in a critical way, so as to advance in a productive understanding of this relationship for research. Different conceptions of semantics and its links with social structures are described and analysed. Along with the above, a possible interrelation between both concepts is questioned, and it is replaced with an approach nearer to system theory and evolutionary theory. The paper concludes with a proposal for the distinction between semantic and social structure, which emphasizes the chances that society describes its possible structures by means of its own semantics. This approach would allow an approach to semantics in an anticipatory, reconstructive and constitutive way with regard to social structures.

KEYWORDS: Social structure; Semantics; Social Systems; Functional differentiation; History of ideas

La diferencia entre estructura social y semántica es una de las distinciones más influyentes de la teoría de sistemas. Tan pronto como las investigaciones teórico-sistémicas se llevan al terreno empírico, éstas se sirven con gusto de dicha distinción, asumiendo una relación entre ambos términos para denominar situaciones, las cuales pueden ser observadas, hasta cierto punto, de manera independiente y luego ser estudiadas en sus interrelaciones. El presente trabajo intenta determinar con mayor profundidad el perfil analítico de esta distinción central. Éste ha surgido con motivo de una

\author{
REVISTA MAD - REVISTA DEL MAGÍSTER EN ANÁLISIS SiSTÉMICO APLICADO A LA SOCIEDAD \\ ISSN 0718-0527 \\ Facultad de Ciencias Sociales, Departamento de Antropología. \\ Universidad de Chile \\ www.revistamad.uchile.cl
}

DOI: 10.5354/0718-0527.2016.42794 
conferencia sobre historia de las ideas y sus alternativas historiográficas, lugar al que pertenece la distinción central entre estructura social y semántica. Los argumentos a continuación se concentrarán en la tarea constructiva de precisar la opción analítica de la distinción entre estructura social y semántica. Por esta razón, el momento crítico de la definición remite a la historia de las ideas. Para comenzar nuestro estudio, mencionaremos dos conjuntos de preguntas relevantes para la discusión actual, de modo de poder dar cuenta y comparar estrategias alternativas de investigación sobre el cambio cultural e intelectual.

1. Hay necesidades analíticas para una descomposición y particularización de las unidades basales del cambio cultural. Estas deben permanecer arbitrariamente pequeñas para que, en el campo de elección de los conceptos, no conduzcan a decidir que el cambio cultural se debe entender como discontinuo y no como continuo. Las unidades elementales pequeñas dejan abierta esta pregunta. Son compatibles con cambios continuos; pero es también imaginable que ciertos impulsos de cambio pequeños se presenten bajo la forma de complejos estrechamente interconectados u oleadas, los cuales harían probables quiebres o discontinuidades en el cambio cultural. Este argumento tiene un trasfondo teórico evolucionista. Supone que es preferible un acercamiento metódico que sea al menos compatible con el pensamiento teórico de la evolución (Stichweh 1999) y que permita tratar, con los mismos instrumentos conceptuales, largas fases de un apenas perceptible cambio cultural, así como también oleadas súbitas de desarrollo. Este problema y sus propuestas de solución han sido introducidos en la teoría de evolución bajo el concepto central de punctuated equilibria (Gould 1989).

En lo que concierne a las ideas, se puede dudar de la capacidad de cumplir con estas condiciones formales y metódicas. Las ideas, a diferencia de la semántica, vale decir, de la alternativa favorecida, parecen depender de una cierta distancia entre sí para que uno esté dispuesto a llamarlas ideas ${ }^{1}$.

2. La teoría de sistemas y la cibernética suponen que la información, la comunicación y la observación son procesos conducidos por diferencias (Shannon \& Weaver 1949; Ruesch \& Bateson 1951; Bateson 1973; Luhmann 1984). Siguiendo estas perspectivas, ¿responden convenientemente las teorías y conceptos al cambio cultural? Esto afecta precisamente a la semántica. Ésta descansa en muchos casos en contra-conceptos asimétricos (Koselleck 1979); emplea antónimos que, en procesos de transformación, se intercambian unos con otros (Holmes 1987); engendra códigos binarios y clasificaciones sociales con lógicas de distinción muy diferentes (Luhmann 1986). Surge nuevamente la pregunta si la tradición de reflexiones

${ }^{1}$ Cf. unit-ideas en La Vopa (1988: 169, et passim). 
Estructura social y semántica: la lógica de una distinción sistémica

sobre las ideas muestra una riqueza parecida en distinciones. Además, si se toma en serio el basamento en diferencias por parte de la observación y la comunicación, se hace inevitable la pregunta sobre las distinciones que sirven de base a los conceptos de idea y semántica. En el primer caso, la distinción entre idea y realidad ${ }^{2}$ sea tal vez una distinción algo estéril que parece insinuar más bien un hiato inconciliable y de ahí decepciones resultantes de la realización de las ideas, como muestra acaso su dinámica de niveles interactivos. En lo que respecta a la semántica, se trata de la distinción central -rica en tensiones- entre estructura social y semántica, la cual no inspira preguntas sobre oportunidades de realización, sino más bien sobre relaciones fundamentales y direcciones de atribución. A causa de justamente las ambigüedades de esta última distinción, las dificultades que presenta su uso y las oportunidades de conocimiento que ella ofrece, van tras sus pasos las reflexiones siguientes.

II

La distinción entre estructura social y semántica debe su actual relevancia a la teoría de sistemas de Niklas Luhmann, quien la desarrolló de forma paralela a los influyentes intentos de las ciencias históricas en la búsqueda de una semántica histórica ${ }^{3}$. Se debe también destacar su paralelismo y competencia con una de las distinciones clásicas de la sociología: cultura vs estructura social. Como es sabido, no es nada sencillo decir que es en realidad cultura (Luhmann 1995; Stichweh 1999), sobre todo si uno intenta aferrarse a ambos lados de la distinción entre cultura y estructura social y evitar así la práctica común de sociólogos y antropólogos culturales de incorporar en el sentido de uno de los lados del concepto, al amplio espacio de significado del otro ${ }^{4}$. Por esto, será necesario examinar si una dificultad parecida se repite en el caso de la relación entre estructura social y semántica.

Si se mira el trabajo de Niklas Luhmann, uno se encuentra con varias versiones para el concepto de semántica que no son congruentes entre sí. Así, se habla de "formas de una sociedad" en contraposición a los acontecimientos de acción y vivencia (Luhmann 1980: 19). En esta versión, la semántica pone a disposición formas que pueden ser capturadas durante los acontecimientos de la vivencia y la acción. En el mismo texto de 1980, Luhmann señala también que la semántica es "sentido altamente generalizado y relativamente independiente de la situación que se encuentra

\footnotetext{
${ }^{2}$ Véase Weischedel (1961) para el ejemplo de una institución (universidad), de la cual se dice a menudo que refiere a una idea, todo lo cual ha llevado a Cohen y March (1974) a señalar que prácticamente cada miembro de una universidad puede dar una conferencia sobre la idea de universidad, pero casi nadie querría escuchar dicha conferencia.

${ }^{3}$ Documentada voluminosamente en el diccionario enciclopédico Conceptos históricos básicos (Brunner, Conze \& Koselleck) y el Manual de conceptos político-sociales básicos en Francia 1680-1820 (Reichardt, Gumbrecht \& Chartier).

${ }^{4}$ Véase Durham (1991: 30, 182s.) como ejemplo de una teoría antropológico-cultural, la cual cree contar con bastantes conceptos residuales de estructura social (en este caso: desigualdades de poder).
} 
disponible" (Luhmann 1980: 19). Como sabemos, el uso del sentido es universal. Toda vivencia, acción y comunicación descansa en su uso. Tan pronto como el sentido empleado se despega del carácter concreto de las situaciones, se trataría de semántica. En el libro "sistemas sociales" de 1984, Luhmann añade una tercera formulación que habla de una "provisión de temas posibles" reservados para la comunicación ${ }^{5}$. Simultáneamente se acentúa el momento en que Luhmann identifica la semántica con comunicación textual, fijada y protegida por escrito. Lo último no me parece una relación inevitable o convincente. En último término, en sociedades ágrafas se pueden observar también amplias generalizaciones de sentido. El propio Luhmann indica en un lugar que aquellas formulaciones dobles siempre repetitivas, las cuales están presentes en la comunicación impresa a través del siglo XVIII, como por ejemplo "la magnificence et estat que peut tenir un prince", serían remanentes de requisitos propios de la comunicación oral "de consolidar la impresión por medio de la repetición y la insistencia" (Luhmann 1989: 82). En estos casos, se puede ver cómo se marca algo como semántica por medio de la repetición explícita. En este sentido, se puede suponer una semántica de sociedades ágrafas y formas específicas de semántica en sociedades con escritura. A pesar de esta reserva, parece que es mucho más sencillo producir semánticas que son puestas a disposición deliberadamente y sobre todo que varían, cuando es posible recurrir a la escritura.

Otro componente llamativo de la comprensión de Luhmann es que menciona una y otra vez semánticas "cultivadas" y "dignas de ser conservadas". Se admite así una valoración positiva y -algo insólito en Luhmannse afirma teóricamente que las sociedades se dan parte de su propia semántica. Tampoco ésta parece ser una relación inevitable. A fin de cuentas, hay argumentos a favor de la existencia de una semántica popular o revolucionaria y para la posibilidad de su éxito en la sociedad. Más aún, uno puede preguntarse si es o no probable que ciertos excluidos tengan éxito en subvertir puntualmente la semántica de una sociedad (Luhmann 1997: 538s), con los cual conseguirían un reingreso socio-estructural.

En la última fase del pensamiento de Luhmann domina una última versión que relaciona la semántica con el concepto de observación. La semántica permite y conduce la auto-observación y auto-descripción de la sociedad, pues provee de distinciones a estas operaciones (Luhmann 1997: 538s). En este sentido, la semántica es una provisión de distinciones.

¿Cómo se construye el contra-concepto de "semántica", esto es "estructura social"? En sus trabajos sobre semánticas históricas, Luhmann

\footnotetext{
5 “Debe haber, entonces, un requerimiento que sirva de mediador entre interacción y lenguaje -una especie de provisión de posibles temas listos para una entrada súbita y rápidamente comprensible en procesos comunicacionales concretos. Llamamos a esta provisión de temas, cultura, y cuando esta se ha almacenado especialmente para fines comunicativos, semántica. La semántica es digna de conservarse y, por lo tanto, es una parte de la cultura, cuando nos transmite la historia de los conceptos y las ideas." (Luhmann 1984: 224)
} 
Estructura social y semántica: la lógica de una distinción sistémica

se refiere a las estructuras sociales sobre todo como formas de diferenciación sistémica - diferenciación segmentaria, centro/periferia, estratificación y diferenciación funcional. Éstas parecen personificar una realidad estructural dura que puede ser pensada como independiente a la semántica y ser puesta precisamente por eso en una relación de correspondencia con desarrollos semánticos. Sin embargo, si uno no se concentra en estas pocas grandes estructuras en la evolución de la sociedad (es decir, el extremadamente raro cambio exitoso en la forma de diferenciación de la sociedad) y presta atención en su lugar más bien al fenómeno general de la estructura social, la diferencia entre estructura social y semántica no queda tan clara. Como es sabido, Luhmann describe con alguna consistencia a las estructuras de los sistemas sociales como estructuras de expectativas ${ }^{6}$. Si se comprende a las expectativas como formadoras de estructuras, éstas no sería entonces otra cosa que sentido altamente generalizado y sería muy difícil distinguirlas de la semántica, pues ésta tendría que ser entendida como sustancial para la formación de estructuras sociales.

Esto lleva a las ideas de Niklas Luhmann a un problema. En sus libros sobre semánticas históricas, menciona repetidamente una correlación entre estructura de la sociedad y semántica, pero de vez en cuando actúa como si la pregunta sobre esta correlación fuese el verdadero programa de explicación de estos estudios (Luhmann 1980). Sin embargo, sólo se puede hablar de correlaciones cuando las situaciones en cuestión no se encuentran vinculadas por lazos primordiales unilaterales o mutuos y por esta razón se debería renunciar a hablar de correlación para el caso aquí discutido sobre la relación entre estructura social y semántica. Sólo se podría evitar la renuncia a esta tan atractiva manera de hablar en ciencias sociales respecto de una correlación entre estructura social y semántica, si se asume que las estructuras sociales se encuentran libres de sentido y distinciones. Sin embargo, esta opción está vedada por principio a una teoría de sistemas que ha decidido que el sentido es el modo de operación de sistemas síquicos y sociales, y para una teoría que ubica a la comunicación como base de lo social.

En cuanto a las macro-estructuras de la diferenciación de la sociedad, también se debe renunciar a hablar en términos de correlaciones. La diferenciación funcional es inimaginable sin sus semánticas de diferenciación funcional. Éstas identifican, en la ejecución de las operaciones, las fronteras de los sistemas funcionales mediante distinciones manejables, las cuales reproducen la lógica de la diferenciación funcional. Luhmann mismo señala en ocasiones que no se puede reconocer al sistema político en el nivel operativo del uso del poder, dado que éste ocurre en muchos sistemas funcionales, desde la educación hasta la comunicación íntima. Por esto se requiere de la semántica del Estado, para la identificación de las fronteras

\footnotetext{
${ }^{6}$ Más detalladamente en Luhmann (1984).
} 
del sistema político, la cual acompaña a todas las operaciones como remisión de sentido, haciendo posible distinguir al suceso político de las muchas formas de uso del poder que no refieren al Estado (Stäheli 1998: 316).

\section{III}

La renuncia a una determinada versión fuerte de la distinción e interrelación entre estructura social y semántica no acarrea el colapso total de dicha distinción. Solamente significa que las interrelaciones se multiplican y complican. Esbozaré a continuación algunas de estas relaciones.

1. Una primera forma de relación entre estructura social y semántica se puede designar bajo el concepto de diferenciación. Respecto de ésta, se deben distinguir nuevamente dos aspectos distintos. Por un lado, una ventaja del concepto de diferenciación radica precisamente en que permite evitar la división entre estructura social y semántica. $\mathrm{Si}$, por ejemplo, se trata de la diferenciación del derecho, nos referimos no solamente a la diferenciación de los aspectos profesionales y organizativos del sistema legal (la profesión jurídica, los juicios, etc.), sino también a la terminología, la dogmática y la teoría legal -todo esto como parte del mismo sistema ${ }^{7}$. Por otro lado, un proceso concreto de diferenciación puede caracterizarse precisamente por emancipar sus procesos de producción de semánticas, despegándolos de referencias y expectativas de resultados sociales. Esta es una de las implicaciones importantes del concepto de discurso. Un discurso es, en términos generales, un sistema de producción emancipada de semántica que circula en sí mismo, pero que no puede darse fin a sí mismo, y que depende más bien de puntos de apoyos externos que conectan con el discurso o le retiran sus condiciones. En lugar del concepto de correlación, se pueden incorporar conceptos teórico-sistémicos como acoplamiento estructural para dar cuenta de las relaciones exteriores y las influencias causales de un sistema semántico. Los entramados diferenciados de producción semántica -que podrían analizarse perfectamente como sistemas sociales- pueden contraer evolutivamente relaciones de acoplamiento estructural con otros sistemas sociales, irritándolos con su semántica y recibiendo perturbaciones en su auto-reproducción. La diferenciación es un suceso semántico y socio-estructural, se extiende, pero también divide. Una formulación conveniente para lo que significa la distinción entre estructura social y semántica podría ser que ésta es una variable histórica, cuya manifestación concreta depende de procesos del diferenciación.

2. Este análisis que entiende la diferenciación como un proceso extensivo a la estructura social y la semántica como parte del mismo sistema, o como

\footnotetext{
${ }^{7}$ Véase esta idea de diferenciación en Luhmann (1980: 20s.; Luhmann 1997: 538s.; Stichweh 1984).
} 
Estructura social y semántica: la lógica de una distinción sistémica

un proceso de división de sistemas en subsistemas, lleva a otro tipo de relación que también se relaciona con una interpretación del concepto de discurso. Foucault señala en varios lugares a los discursos como dispositivos (Foucault 1978). El término refiere a discursos que, en determinado punto, se traducen en decisiones, órdenes o formaciones (militares) (p. ej., las de un Señor), las cuales dependen de precauciones socio-estructurales que apoyen esta aplicación o la exijan. En este sentido, en dichos casos no puede tratarse de discursos emancipados. Su grado de autonomía cognitiva permanece limitado. Un discurso que se convierte en dispositivo da pie evidentemente a entramados de relaciones de acción social, pues las distinciones que regulan este discurso conducen directamente a una praxis de acción, la cual se conduce semánticamente en las ejecución de sus actos individuales. En esta versión de la distinción entre estructura social y semántica, manejamos la variante más punzante de todas las interpretaciones de la semántica, según la cual la semántica es sustancialmente responsable de la formación de estructuras en un sistema social. Las expectativas que forman estructuras no cristalizan accidentalmente en los entramados comunicacionales de un sistema, para luego sistematizarse en semánticas; ellas se derivan más bien, como si se tratase de instrucciones, de una semántica.

3. Una tercera variante de la relación entre estructura social y semántica refiere nuevamente al rol adelantado o anticipatorio de la semántica. Para describir esta situación se pueden usar provechosamente conceptos teórico-evolutivos. Niklas Luhmann usa el concepto, familiar para los evolucionistas, de preadaptative advances, con el cual se designan invenciones en nuestro caso, invenciones semánticas- que, si bien en un principio se ajustan provisoriamente a un sistema social, no obstante sobreviven, pero sólo a posteriori adquieren una función social, la cual no estaba prevista cuando la invención (semántica) hizo su aparición ${ }^{8}$. Esta función posterior quizá nunca se habría producido si no hubiese aparecido esta invención para acompañar sugestivamente su proceso de formación. La semántica parece tener un carácter instructivo también en este modelo, pero no se crea con dicho propósito, se ve sorprendida cuando surge la función, al igual que el sistema social donde ésta se lleva a cabo.

Un ejemplo histórico que permite aclarar este modelo explicativo lo ofrece la semántica de la libertad académica, con sus componentes de libertad de enseñanza y libertad de aprendizaje, la cual adquirió gran notoriedad en las universidades alemanas entre los siglos XVII y XVIII (Stichweh 1991, 1994: Cap. 13). Esta versión temprano-moderna de libertad académica se vio favorecida, entre otras cosas, por cálculos económicos estatales,

\footnotetext{
${ }^{8}$ Véase Luhmann (1978). Un término distinto han propuesto Stephen J. Gould e Isabel S. Vrba para estas situaciones -que por su parte han aconsejado llamar preadaptation- es exaptation (véase Gould 1977: 434, Fn. 3; Gould 1982; Gould \& Vrba 1982). Ejemplos interesantes sobre exaptation tomados de la biología se encuentran en Zimmer (1998: 37s.).
} 
de acuerdo con los cuales las universidades debían desarrollar fuerzas de atracción sobre las fronteras de pequeños territorios alemanes singulares. Por este motivo, las universidades se llenaban de estudiantes venidos desde otros territorios y que aportaban "dinero al país", todo lo cual afectó de manera inusitada a las libertades concernientes a la organización de los estudios, pero también a las maneras de vivir. Lo mismo ocurrió con la libertad de enseñanza de los docentes, la cual hizo posible reclutar profesores más allá de las fronteras locales y así, debido a que la universidad se hacía cada vez más atractiva para sus profesores, las universidades pudieron contar con profesores de renombre, todo lo cual aumentó aún más la fuerza de atracción hacia los estudiantes de lugares más lejanos. Se acepta usualmente el supuesto que la libertad académica fue una de las libertades corporativas típicas del Ancien Régime que luego se disolvió junto con la caída de dicha forma social. Pero, en efecto, la libertad académica probó ser en este sentido un avance preadaptativo, al recaer sobre la universidad basada en el imperativo de la investigación del siglo $\mathrm{XIX}^{9}$ servicios de fundamentación y además una infraestructura relevante, todo lo cual no podía haberse previsto en el siglo XVIII. Luego la libertad académica de la universidad alemana se convirtió en un correlato de suyo evidente para esta nueva universidad orientada a la investigación y para los patrones de roles de los profesores y estudiantes, a pesar de que ésta no hubiese sido inventada en absoluto para dicho propósito.

Los avances preadaptativos, en este sentido preciso, son un caso relativamente especial. Un modelo más general, que se encuentra frecuentemente en la literatura sociológico-cultural, es la idea que la semántica tiene preparada para la sociedad un variety pool consistente de esbozos de posibilidad alternativos para realizaciones socio-estructurales. En cuanto a la forma y composición dicho variety pool, se pueden observar múltiples modelos y propuestas (Stichweh 1999; Parsons 1961, 1973).

Otra interesante idea, proveniente también de la teoría de la evolución, habla de polimorfismos. Con ella se señala que dentro de un semántica hay una población de conceptos con significados y valoraciones divergentes entre sí, los cuales abren enlaces muy distintos, según sea la variante elegida dentro de dicha población. El teórico de la ciencia David Sloan Wilson, ejemplifica esto a partir la semántica inglesa del término selbst que, a partir de conceptos como self-interest, selfish, selfless, selfhood, etc., mezcla valoraciones positivas y negativas, dando espacio así a posibilidades de enlace diametralmente contradictorias (Wilson 1990).

4. Un cuarto tipo de relación posible entre estructura social y semántica va en la dirección contraria. Acentúa la retroactividad [Nachträglichkeit] de la

\footnotetext{
9 Sobre este imperativo de investigación y las reformas estructurales de la universidad, véase Turner
} (1973). 
Estructura social y semántica: la lógica de una distinción sistémica

semántica. Para Niklas Luhmann, esta parece ser la comprensión dominante: una nueva forma de realidad pudo haberse establecido únicamente, si anteriormente ésta fue provista de una semántica adecuada de observaciones y descripciones. También en esta interpretación se debe hacer una corrección. Urs Stäheli lo ha hecho en interesantes trabajos que completan el modelo luhmanniano de la retroactividad con la idea de retroactividad constitutiva (Stäheli 1998, 2000), basándose en una especie de paradigma psicoanalítico. Un escenario interactivo presenta apenas algunos significados; sin embargo, en la medida en que se va completando con observaciones y descripciones retroactivas, crecen sus implicaciones de sentido, las cuales no se pueden formular razonablemente como la mera comprensión de un sentido pensado originalmente, pero que no obstante no se puede impugnar su relación como inapropiada o extraña. Estas implicaciones de sentido son parte del modo en que este escenario interactivo produce enlaces en la comunicación y, en este sentido, son parte de la realidad social que comunican. Es en este sentido que se debe entender en general la función de una semántica histórica retroactiva. También es -a pesar de su retroactividad-fundamental para el acontecimiento que sigue, porque sólo a través de ella se cristalizan los significados que muestran después la relevancia histórica de dicho acontecimiento. Este modelo puede ser puesto a prueba fácilmente en un ejemplo como el de la revolución francesa, dado que sería totalmente imposible entender este evento sin estratos de interpretación que la historia posterior ha puesto sobre él, a pesar que en las ciencias históricas sea imaginable un acceso metódico que lo intente. No obstante, si tuviese éxito, sería en lo sucesivo sólo una de las muchas interpretaciones de la revolución francesa.

5. Otra condición que hace difícil la separación entre estructura social y semántica, y que contribuye al fracaso de la correlaciones, tiene que ver con la historicidad de esta separación y con la historicidad de la distinción que sirve de base. Estructura social y semántica están ligadas por procesos de diferenciación que subyacen a ambas y asimismo son ambas un resultado de la evolución. En sus reflexiones teóricas sobre la evolución, Niklas Luhmann distingue la evolución de la sociedad de la evolución de la semántica, es decir, supone que se requieren dos teorías diferentes, pero formalmente emparentadas, para explicar, en un caso, la evolución de las estructuras sociales y, en el otro, la evolución de estructuras semánticas ${ }^{10}$. Este es un postulado plausible para sociedades estratificadas y para las altas culturas tradicionales, donde una sociedad compuesta de colectivos sociales todavía se cierra bajo la forma de estratos sociales respecto de todas las diferencias factuales de la comunicación y explica al mismo tiempo que hay también un sistema semántico cerrado, el cual está diferenciado en segunda instancia por temáticas factuales específicas. Una pregunta decisiva al respecto es,

${ }^{10}$ Sobre esto y en lo sucesivo Luhmann (1997: esp. Cap. 3, X-XI). 
siguiendo este punto de vista, ¿qué es lo que cambia en un sistema de sociedad diferenciado funcionalmente? Si se mira desde un punto de vista socioestructural, la evolución tiene lugar precisamente en el nivel de los sistemas funcionales, es decir, debe ser descrita como evolución del derecho, de la economía, de la ciencia, etc. Con esto se disuelve también la cultura uniforme de la semántica de la vieja Europa. La producción semántica tiene lugar primero dentro de los sistemas funcionales. ¿Esto sugiere también que se debería suponer además una evolución independiente de cada semántica en cada sistema funcional? Luhmann deja expresamente abierta esta pregunta en La sociedad de la sociedad. Pero una respuesta posible puede ser que esto no sólo heriría principios elementales de teoría económica, sino que tampoco sería esclarecedor factualmente, pues contradiría la cercanía de la estructura social y la semántica con el concepto de expectativa. Esto conduciría a la conclusión de que la teoría de la evolución del sistema respectivo tiene que ser una teoría de la evolución de su estructura social y de su semántica, con conclusiones correspondientes sobre la (in)divisibilidad de estructura social y semántica.

6. En casi todos los sistemas funcionales hay conocimiento en abundancia. Éste se distingue por las condiciones de su corrección. Los conocimientos de la semántica, por su parte, se distinguen porque pueden no ser correctos o ser equivocados. En cuanto a la semántica de las auto-descripciones de los sistemas funcionales, hay una llamativa asimetría entre éstos. En el caso de la ciencia, su auto-descripción (es decir la teoría científica) usa el medio de comunicación del sistema (Stäheli 1998: 327s., 2000: 211s.). Ella produce comunicaciones como teoría científica, las cuales son posibles de codificar como verdad y las presenta bajo la forma de publicaciones científicas. De este modo, desaparecen en cierto modo en las actuaciones normales del sistema. Sin embargo, no ocurre lo mismo con otras auto-descripciones de sistemas funcionales (como la teoría legal o la teoría económica). Si bien éstas se refieren al medio y las operaciones del sistema respectivo, su estatus propio permanece incierto. ¿Se trata de comunicaciones científicas? ¿Participan en el medio de la verdad del sistema científico? ¿Y qué se deriva de esto para la interrelación entre estructura social y semántica? Una situación que podemos indicar con esta observación es que la pregunta sobre la clausura operativa del sistema puede presentarse de modo distinto para la estructura social y la semántica, y que también este es un momento que distingue entre estructura social y semántica. Antes me referí a la tesis que el sistema político, en el nivel operativo del uso del poder, no puede pensarse de manera convincente como sistema cerrado, pues para la clausura operativa es necesaria la ejecución paralela de la semántica del Estado a través de las comunicaciones del poder. En el caso que aquí discutimos, nos encontramos en cierto modo con el fenómeno inverso. Determinados sistemas funcionales -el derecho o la economía- se deben reconocer en el nivel 
Estructura social y semántica: la lógica de una distinción sistémica

de sus operaciones -decisiones de acuerdo con criterios legales, entramados de pagos- como sistemas funcionales operativamente clausurados. Sin embargo, éstos producen una semántica en sus propias auto-descripciones -teoría legal, teoría económica- que recurren a acoplamientos estructurales con la ciencia y que, en casos puntuales, no se puede decidir en qué sistema funcional se ubican estas comunicaciones. En estos casos, la semántica no es un factor de protección de los límites del sistema (como ocurre justamente en el ejemplo del sistema político), sino que opera como un factor de multiplicación e indeterminación de los límites de los sistemas.

7. Una última reflexión que podemos introducir aquí concierne la pregunta de la latencia de la semántica. Se ha explicado que la semántica es fundamental para la formación de estructuras y la operación de los sistemas sociales, pues provee a la comunicación de distinciones y expectativas, sin las cuales nada se podría comunicar. ¿Puede permanecer latente la semántica?, es decir, que ésta nunca operaría en las comunicaciones (tampoco implícitamente), y sin embargo sería reactualizable. Entonces su presencia estaría limitada aparentemente a textos leídos sólo ocasionalmente o casi olvidados, pero esto implicaría que no es una semántica de la sociedad donde se presenta en estado de latencia. Según esta interpretación, la semántica no podría distinguirse de la cultura, dado que este momento de latencia y larga duración se atribuye típicamente al concepto de cultura (Stichweh 1999). Esto nos sugiere evitar la noción de una semántica latente $\mathrm{y}$, en lugar de esto, sostener que la semántica opera siempre en la actualidad como la semántica de una sociedad observada ${ }^{11}$. De este modo, como hemos tratado de señalar, la semántica es de muchas maneras constitutiva, anticipativa y/o retroactiva en relación con la sociedad donde actúa. Se puede usar el análisis de las semánticas para descomponer la estructura social, pues se trata de entenderlas en las investigaciones. Pero sólo en este sentido se puede hablar de una correlación entre estructura social y semántica. De esto se deriva que nunca se puede sostener que un análisis sea solamente semántico, separado de realidades fundamentales.

En las reflexiones precedentes se hizo patente el espectro de prestaciones que pueden otorgar los análisis semánticos, para volver al punto de partida del presente texto, y al mismo tiempo destacamos que el potencial analítico de un análisis semántico es más provechoso que la mera historia de las ideas, la cual no puede pretender un alcance comparable al mundo social actual producido por operaciones comunicativas reales.

\footnotetext{
${ }^{11}$ Sólo se puede hablar de latencia en este sentido, si se comprende la semántica como preadaptive advance, es decir, como si contuviese potenciales de evolución sucesiva que son atribuidos retrospectivamente.
} 


\section{IV}

Las propuestas de corrección aquí presentadas para el manejo de la distinción entre estructura social y semántica introducen de mejor manera la distinción en la teoría de sistemas. En el lugar del concepto de correlación, para el cual no hay interpretaciones convincentes en la teoría de sistemas, se introducen nociones teórico-sistémicas centrales como diferenciación y acoplamiento estructural. En el lugar de una comprensión casi ontológica de estructura social y semántica que, siguiendo a Luhmann, haría necesaria una tercera variable -como el concepto de complejidad-, se propone una variabilidad histórica y situacional de la distinción. Tanto la estructura social como la semántica tienen que ver con sentido altamente generalizado que va más allá de la situación puntual de distinción y comunicación. Bajo estas condiciones, una versión plausible de la distinción quizá sea que una sociedad describe las estructuras posibles para dicha sociedad mediante su semántica. Una semántica así entendida puede comportarse de modo anticipativo, reconstructivo y constitutivo para las estructuras sociales. Lo que distingue a la semántica de las estructuras sociales es que el sentido, que está generalizado en semánticas y que abarca posibles expectativas en diversas situaciones específicas, opera allí de un modo relativamente inespecífico en cuanto a la distinción entre expectativas cognitivas y normativas. La determinación de si las estructuras de las expectativas son empavesadas como normativas o cognitivas, se efectúa en los procesos mismos de formación de estructuras, respecto de los cuales la cultura semántica de una sociedad toma distancia, precisamente para no tener que participar ella misma en la conducción que determina si éstas serán normativas o cognitivas.RM

\section{REFERENCIAS}

Bateson, G. (1973). Steps to an Ecology of Mind. Collected Essays in Anthropology, Psychiatry, Evolution and Epistemology. London: Paladin Books.

Cohen, M. \& March, J. (1974). Leadership and Ambiguity: The American College President. New York: McGraw-Hill.

Durham, W. H. (1991). Coevolution. Genes, Culture, and Human Diversity. Stanford: Stanford University Press.

Foucault, M. (1978). Was ist Kritik? Berlin: Merve.

Gould, S. (1977). Ontogeny and Phylogeny. Cambridge Mass: Harvard University Press.

Gould, S. (1982). Darwinism and the Expansion of Evolutionary Theory. Science, 216(4544), 380-387.

Gould, S. (1989). Punctuated Equilibria in Fact and Theory. Journal of Social and Biological Structures, 12(2-3), 117-136.

Gould, S. \& Vrba, E. (1982). Exaptation - A Missing Term in the Science of Form. Paleobiology, 8(1), 4-15.

Holmes, S. (1987). Poesie der Indifferenz. In D. Baecker, J. Markowitz, R. Stichweh, H. Tyrell, \& H. Willke (Eds.), Theorie als Passion. Niklas Luhmann zum 60. Geburtstag (pp. 15-45). Frankfurt aM: Suhrkamp.

Koselleck, R. (1979). Zur historisch-politischen Semantik asymmetrischer Gegenbegriffe. In Vergangene Zukunft (pp. 211-259). Frankfurt aM: Suhrkamp. 
Estructura social y semántica: la lógica de una distinción sistémica

La Vopa, A. (1988). Grace, Talent and Merit. Poor Students, Clerical Careers, and Professional Ideology in Eighteenth- century Germany. Cambridge Mass: Cambridge University Press.

Luhmann, N. (1978). Geschichte als Prozeß und die Theorie soziokultureller Evolution. In Soziologische Aufklärung 3 (pp. 178-197). Opladen: Westdeutscher Verlag.

Luhmann, N. (1980). Gesellschaftliche Struktur und semantische Tradition. In Gesellschaftsstruktur und Semantik. Studien zur Wissenssoziologie der modernen Gesellschaft, Bd. 1 (pp. 9-71). Frankfurt aM: Suhrkamp.

Luhmann, N. (1984). Soziale Systeme: Grundriß einer allgemeinen Theorie. Frankfurt aM: Suhrkamp.

Luhmann, N. (1986). „Distinctions directrices": Über Codierung von Semantiken und Systemen. In F. Neidhardt, R. Lepsius, \& J. Weiß (Eds.), Kultur und Gesellschaft. Sonderheft 27 der KZfSS (pp. 145-161). Opladen: Westdeutscher Verlag.

Luhmann, N. (1989). Staat und Staatsräson im Übergang von traditionaler Herrschaft zu moderner Politik. In Gesellschaftsstruktur und Semantik. Studien zur Wissenssoziologie der modernen Gesellschaft, Bd. 3 (pp. 65-148). Frankfurt aM: Suhrkamp.

Luhmann, N. (1995). Kultur als historischer Begriff. In Gesellschaftsstruktur und Semantik. Studien zur Wissenssoziologie der modernen Gesellschaft, Bd. 4 (pp. 3154). Frankfurt aM: Suhrkamp.

Luhmann, N. (1997). Die Gesellschaft der Gesellschaft, Bd. 1-2. Frankfurt aM: Suhrkamp.

Parsons, T. (1961). Culture and the Social System - Introduction. In T. Parsons, E. Shils, K. Naegele, \& J. Pitts (Hrsg.), Theories of Society. Foundations of Modern Sociological Theory (pp. 963-993). New York: The Free Press of Glencoe.

Parsons, T. (1973). Culture and Social System Revisited. In L. Schneider \& C. Bonjean (Eds.), The Idea of Culture in the Social Sciences (pp. 33-46). Cambridge Mass: Cambridge University Press.

Ruesch, J. \& Bateson, G. (1951). Communication: The Social Matrix of Psychiatry. New York: W. W. Norton \& Company.

Shannon, C. \& Weaver, W. (1949). The Mathematical Theory of Communication. Urbana Ill: University of Illinois Press.

Stäheli, U. (1998). Die Nachträglichkeit der Semantik. Zum Verhältnis von Sozialstruktur und Semantik. Soziale Systeme, 4(2), 315-339.

Stäheli, U. (2000). Die Operativität von Selbstbeschreibungen. Gesellschaftsstruktur und Semantik. In Sinnzusammenbrüche. Eine dekonstruktive Lektüre von Niklas Luhmanns Systemtheorie (pp. 184-223). Weilerswist: Velbrück.

Stichweh, R. (1984). Zur Entstehung des modernen Systems wissenschaftlicher Disziplinen. Physik in Deutschland 1740- 1890. Frankfurt aM: Suhrkamp.

Stichweh, R. (1991). Der frühmoderne Staat und die europäische Universität. Zur Interaktion von Politik und Erziehungssystem im Prozeß ihrer Ausdifferenzierung (16.-18. Jahrhundert). Frankfurt aM: Suhrkamp.

Stichweh, R. (1994). Wissenschaft, Universität, Professionen: Soziologische Analysen. Frankfurt aM: Suhrkamp.

Stichweh, R. (1999). Kultur, Wissen und die Theorien soziokultureller Evolution. Soziale Welt, 50(4), 459-470.

Turner, R. S.(1973). The Prussian Universities and the Research Imperative, 1806-1848. Princeton: Princeton University.

Weischedel, W. (Ed.). (1960). Idee und Wirklichkeit einer Universität: Dokumente zur Geschichte der Friedrich-Wilhelms- Universität zu Berlin. Berlin: de Gruyter.

Wilson, D. (1990). Species of Thought: A Comment on Evolutionary Epistemology. Biology and Philosophy, 5(1), 37-62. 
Zimmer, C. (1998). At the Water's Edge. Macroevolution and the Transformation of Life. New York: Free Press.

SOBRE EL AUTOR

Rudolf Stichweh es actualmente profesor en la Universidad de Bonn, Alemania. Entre sus áreas de especialización se encuentran: teoría sociológica, teoría de sistemas, teoría de la sociedad mundial, sociología de la ciencia y de las universidades, evolución sociocultural, sociología económica, sociología del extranjero y macrosociología histórica. Entre sus últimas publicaciones más destacadas se encuentran: Der Fremde: Studien zu Soziologie und Sozialgeschichte (Suhrkamp, 2010) e Inklusion und Exklusion. Analysen zur Sozialstruktur und sozialen Ungleich heit (junto a Paul Windolf) (VS Verlag für Sozialwissenschaften, 2009).

CONTACTO

Universität Bonn

Forum Internationale Wissenschaft

Heussallee 18-24

D- 53113 Bonn rstichweh@yahoo.de

Recibido: mayo 2016

Aceptado: junio 2016 